\title{
Cavity-induced exciton localization and polariton blockade in two-dimensional semiconductors coupled to an electromagnetic resonator
}

\author{
Emil V. Denning $\odot,{ }^{1,2,3, *}$ Martijn Wubs $\odot,{ }^{1,2,4}$ Nicolas Stenger, ${ }^{1,2,4}$ Jesper Mørk $\odot,{ }^{1,2}$ and Philip Trøst Kristensen $\circledast^{1,2}$ \\ ${ }^{1}$ Department of Photonics Engineering, Technical University of Denmark, 2800 Kgs. Lyngby, Denmark \\ ${ }^{2}$ NanoPhoton - Center for Nanophotonics, Technical University of Denmark, Ørsteds Plads 345A, DK-2800 Kgs. Lyngby, Denmark \\ ${ }^{3}$ Nichtlineare Optik und Quantenelektronik, Institut für Theoretische Physik, Technische Universität Berlin, 10623 Berlin, Germany \\ ${ }^{4}$ Center for Nanostructured Graphene, Technical University of Denmark, 2800 Kgs. Lyngby, Denmark
}

(Received 26 March 2021; revised 13 January 2022; accepted 14 January 2022; published 17 February 2022)

\begin{abstract}
Recent experiments have demonstrated strong light-matter coupling between electromagnetic nanoresonators and pristine sheets of two-dimensional semiconductors, and it has been speculated whether these systems can enter the quantum regime operating at the few-polariton level. To address this question, we present a microscopic quantum theory for the interaction between excitons in a sheet of two-dimensional material and a localized electromagnetic resonator. We find that the light-matter interaction breaks the symmetry of the otherwise translation-invariant system and thereby effectively generates a localized exciton mode, which is coupled to an environment of residual exciton modes. This dissipative coupling increases with tighter lateral confinement, and our analysis reveals this to be a potential challenge in realizing nonlinear exciton-exciton interaction. Nonetheless, we predict that polariton blockade due to nonlinear exciton-exciton interactions is well within reach for nanoresonators coupled to transition-metal dichalcogenides, provided that the lateral confinement can be sufficiently tight to make the nonlinearity overcome the polariton dephasing caused by phonon interactions.
\end{abstract}

DOI: 10.1103/PhysRevResearch.4.L012020

Interfacing an electromagnetic resonator with the spatially extended excitons in a pristine sheet of two-dimensional (2D) semiconductor has recently been demonstrated as a way of obtaining very large Rabi splittings, up to the order of $100 \mathrm{meV}[1-8]$. These strong interactions are of great interest due to the prospect of realizing polaritonic devices [9] such as squeezed-light sources [10,11], polariton lasers [12], and polariton blockade which, in turn, enables the construction of single-photon sources [13-16] and few-photon logic gates [17-19]. Despite a growing interest in these systems, there is a lack of microscopic modeling of the experiments, which are colloquially analysed by use of phenomenological coupledoscillator models. Even if these models can be well fitted to experimental data, the lack of a microscopic foundation limits their ability to predict quantum optical figures of merit of practical interest, such as second-order correlation functions.

In this Letter, we develop a microscopic quantum theory of 2D semiconductors coupled to electromagnetic resonators, which consistently links important dynamical quantitiessuch as the exciton-resonator coupling strength and effective nonlinear exciton-exciton interaction - to fundamental material parameters. The theory applies equally to plasmonic resonators [1-8] and dielectric nanocavities [20-22], includ-

\footnotetext{
*emvo@fotonik.dtu.dk

Published by the American Physical Society under the terms of the Creative Commons Attribution 4.0 International license. Further distribution of this work must maintain attribution to the author(s) and the published article's title, journal citation, and DOI.
}

ing a new generation of dielectric cavities with extreme confinement of light [23-25]. Our approach is based on 2D Wannier-Mott excitons with discrete translation invariance broken only by interactions with the localized electromagnetic resonator, as illustrated in Fig. 1(a). We find that the symmetry-breaking light-matter interaction generates a collective localized exciton mode-an exciton reaction coordinate - defined by the profile of the resonant electric field. The exciton reaction coordinate, in turn, is coupled to an environment of residual exciton modes with a spectral density that depends on the lateral extent of the resonant field. Coupling to the residual exciton modes is pronounced only for very tight lateral confinement of the electric field and can be neglected for confinement lengths above a few nanometers in realistic systems, as illustrated Fig. 1(b). The effective nonlinear exciton-exciton interaction strength in the reaction coordinate also increases with decreasing lateral mode area, thereby making structures with tight lateral confinement crucial for realizing polaritonic devices. Intriguingly, we find that there exists a regime where the lateral confinement is tight enough to enable polariton blockade operation, yet sufficiently large to avoid coupling to residual excitons. In this regime, therefore, one can unambiguously interpret the dynamics in terms of nonlinear multiexciton interactions within the single reaction coordinate. As a main result, we predict that polariton blockade can be reached using a monolayer transition-metal dichalcogenide coupled to an electromagnetic resonator, since the nonlinearity can exceed the dephasing caused by thermal phonon interactions. In contrast to earlier work in the context of quantum wells [13] and 2D materials [26], the present microscopic model is based 

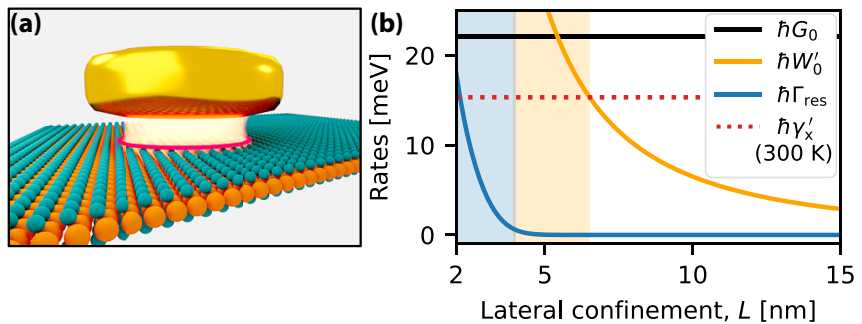

FIG. 1. (a) Illustration of an electromagnetic resonator coupled to a sheet of 2D material. (b) Exciton-resonator coupling $G_{0}$, nonlinear exciton-exciton interaction $W_{0}^{\prime}$ and decay rate into residual exciton modes $\Gamma_{\text {res }}$ as a function of the lateral confinement $L$ of the electromagnetic field, as well as the exciton dephasing from phonon scattering, $\gamma_{x}^{\prime}$, in $\mathrm{WS}_{2}$ at a temperature of $300 \mathrm{~K}$. Yellow shading marks the regime where $L$ is large enough that the residual excitons can be ignored and small enough that $W_{0}^{\prime}$ exceeds the polariton dephasing, such that polariton blockade is possible. Blue shading marks the regime where the residual excitons have a non-negligible impact on the dynamics.

on extended exciton momentum-states, thereby connecting all model parameters directly to measurable material properties and identifying the regime where the influence of the residual excitons cannot be neglected. The model accounts for general electromagnetic resonators with nontrivial field distributions, which extends the applicability beyond state-of-the-art work based on ab initio methods [27]. We also note that recent theoretical work has analyzed the possibility of obtaining polariton blockade [28] with trions in two-dimensional semiconductors, although without considering any dephasing mechanisms, which we find to be the most important limitation for polariton blockade in realistic systems.

The excitons in a sheet of 2D semiconductor can be approximated as interacting bosons with annihilation operators $\hat{b}_{\mathbf{k}}$, labeled by the lateral center-of-mass momentum $\mathbf{k}=\left(k_{x}, k_{y}\right)$ [29-32]. We approximate the excitonic wave function $\phi(\mathbf{q})$ as the Wannier-Mott type [33,34], having a form corresponding to the hydrogen ground state with exciton Bohr radius $a_{\mathrm{B}}$; this approach has previously been demonstrated to agree well with detailed numerical calculations [35]. The Hamiltonian of the excitons and the electromagnetic field is split into three parts as $\hat{H}=\hat{H}_{\mathrm{c}}+\hat{H}_{\mathrm{x}}+\hat{H}_{\mathrm{I}}$. The first term, $\hat{H}_{\mathrm{c}}=\hbar \omega_{\mathrm{c}} \hat{a}_{\mathrm{c}}^{\dagger} \hat{a}_{\mathrm{c}}$, represents the free evolution of the electromagnetic field with resonance frequency $\omega_{\mathrm{c}}$ and bosonic annihilation operator $\hat{a}_{\mathrm{c}}$ [36]. The second term, $\hat{H}_{\mathrm{x}}=\sum_{\mathbf{k}} \hbar \omega_{\mathbf{k}} \hat{b}_{\mathbf{k}}^{\dagger} \hat{b}_{\mathbf{k}}+\hat{W}$, is the exciton Hamiltonian with energies $\hbar \omega_{\mathbf{k}}=\hbar^{2} k^{2} /(2 M)+\hbar \omega_{0}$, where $M=m_{\mathrm{e}}+m_{\mathrm{h}}$ is the total exciton mass $\left(m_{\mathrm{e}}\right.$ and $m_{\mathrm{h}}$ denote the effective electron and hole masses) and $\hbar \omega_{0}$ is the exciton energy gap, accounting for the exciton binding energy and the bare band gap. The operator $\hat{W}=\sum_{\mathbf{k k}^{\prime} \mathbf{q}} \hbar W_{\mathbf{k k}^{\prime} \mathbf{q}} \hat{b}_{\mathbf{k}+\mathbf{q}}^{\dagger} \hat{b}_{\mathbf{k}^{\prime}-\mathbf{q}}^{\dagger} \hat{b}_{\mathbf{k}^{\prime}} \hat{b}_{\mathbf{k}}$ accounts for Coulomb-induced exciton-exciton interaction with matrix elements $W_{\mathbf{k k}^{\prime} \mathbf{q}}[31,32]$. The third term is the light-matter interaction Hamiltonian, $\hat{H}_{\mathrm{I}}=\sum_{\mathbf{k}} \hbar\left(g_{\mathbf{k}}^{*} \hat{a}_{\mathrm{c}}^{\dagger} \hat{b}_{\mathbf{k}}+g_{\mathbf{k}} \hat{a}_{\mathrm{c}} \hat{b}_{\mathbf{k}}^{\dagger}\right)$, with coupling strengths [37]

$$
\hbar g_{\mathbf{k}}=-\frac{e_{0}}{m_{0}} \sqrt{\frac{\hbar}{\pi \epsilon_{0} \omega_{\mathrm{c}} a_{\mathrm{B}}^{2} S}} \int d^{2} \mathbf{r} e^{-i \mathbf{k} \cdot \mathbf{r}} \tilde{\mathbf{F}}_{\mathrm{c}}\left(\mathbf{r}, z_{0}\right) \cdot \mathbf{p}_{\mathrm{cv}},
$$

(a)

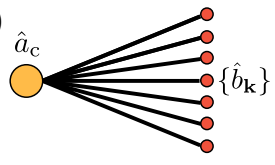

(b)

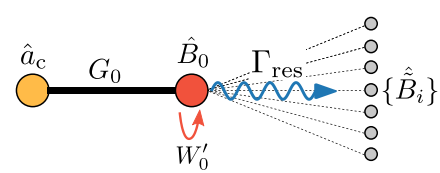

(c)

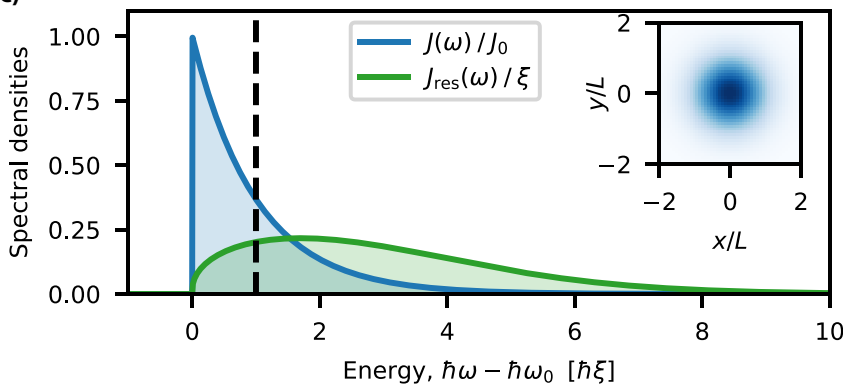

FIG. 2. (a) The localized resonant field ( $\hat{a}_{\mathrm{c}}$, orange dot) is coupled to a continuum of exciton modes with momentum $\mathbf{k}$. (b) Through a linear transformation, the light-matter interaction can be described as a coupling between the resonant field and a single, collective exciton reaction coordinate $\left(\hat{B}_{0}\right)$ which, in turn, is coupled to an environment of residual exciton modes $\left(\hat{\tilde{B}}_{i}\right)$. (c) Exciton spectral density (blue) and residual spectral density (green) for an electromagnetic field with lateral Gaussian confinement corresponding to Eq. (4). The exciton reaction coordinate frequency, $\Omega_{0}$, is indicated with a dashed black line. The inset shows the Gaussian lateral field profile.

where $e_{0}$ is the elementary charge, $m_{0}$ is the free electron mass, $S$ is the surface area of the 2D material sheet located at $z=z_{0}$, and $\mathbf{r}=(x, y)$ is the lateral position. $\tilde{\mathbf{F}}_{\mathrm{c}}(x, y, z)$ is the field profile of the resonant electric field, and $\mathbf{p}_{\mathrm{cv}}$ is the momentum Bloch matrix element. We also include in the formalism the possibility that the fundamental exciton mode is degenerate, as in transition metal dichalcogenides [37].

Through Eq. (1), the interaction Hamiltonian defines the coupling between the resonant electromagnetic field and each of the free excitons, as illustrated in Fig. 2(a). To simplify this model, we can perform a change of basis by defining the collective exciton reaction coordinate with bosonic annihilation operator $\hat{B}_{0}=G_{0}^{-1} \sum_{\mathbf{k}} g_{\mathbf{k}}^{*} \hat{b}_{\mathbf{k}}$, where $G_{0}=\left(\sum_{\mathbf{k}}\left|g_{\mathbf{k}}\right|^{2}\right)^{1 / 2}$. In this way, we can write the light-matter interaction compactly as $\hat{H}_{\mathrm{I}}=\hbar G_{0}\left(\hat{B}_{0}^{\dagger} \hat{a}_{\mathrm{c}}+\hat{B}_{0} \hat{a}_{\mathrm{c}}^{\dagger}\right)$. The collective coupling strength is related to the electric field profile as

$$
G_{0}^{2}=\frac{e_{0}^{2}}{\pi \hbar \epsilon_{0} m_{0}^{2} \omega_{\mathrm{c}} a_{\mathrm{B}}^{2}} \int d^{2} \mathbf{r}\left|\tilde{\mathbf{F}}_{\mathrm{c}}\left(\mathbf{r}, z_{0}\right) \cdot \mathbf{p}_{\mathrm{cv}}\right|^{2} .
$$

Importantly, this coupling strength depends on the field intensity integrated over the entire 2D-material surface. Therefore, in contrast to what one would expect in the dipole approximation [38], tightening the electromagnetic confinement in the lateral direction does not lead to an increased coupling strength, as seen by the horizontal black solid line in Fig. 1, where separability of the field in the lateral and out-of-plane coordinates was assumed. Instead, the overall field strength in the plane of the 2D-material can be quantified through the out-of-plane confinement length scale, $L_{z}=\left(\int d^{2} \mathbf{r}\left|\tilde{\mathbf{F}}_{\mathrm{c}}\left(\mathbf{r}, z_{0}\right)\right|^{2}\right)^{-1}$. Indeed, $L_{z}$ defines an upper bound of the coupling strength through the inequality 
$G_{0}^{2} \leqslant e_{0}^{2}\left|\mathbf{p}_{\mathrm{cv}}\right|^{2} /\left[\pi \hbar \epsilon_{0} m_{0}^{2} \omega_{\mathrm{c}} a_{\mathrm{B}}^{2} L_{z}\right]$, which becomes an equality when the polarization of $\tilde{\mathbf{F}}_{\mathrm{c}}\left(\mathbf{r}, z_{0}\right)$ is aligned with $\mathbf{p}_{\mathrm{cv}}$. In order to implement the change of basis and benefit from the simpler form of $\hat{H}_{\mathrm{I}}$, we must reformulate the noninteracting exciton Hamiltonian $\hat{H}_{\mathrm{x}, 0}:=\sum_{\mathbf{k}} \hbar \omega_{\mathbf{k}} \hat{b}_{\mathbf{k}}^{\dagger} \hat{b}_{\mathbf{k}}$ by defining a new set of exciton modes with bosonic operators $\hat{\tilde{B}}_{i}$ and frequencies $\tilde{\Omega}_{i}$ $(i>0)$, such that it can be written as

$$
\hat{H}_{\mathrm{x}, 0}=\hbar \Omega_{0} \hat{B}_{0}^{\dagger} \hat{B}_{0}+\sum_{i>0}\left[\hbar \tilde{\Omega}_{i} \hat{\tilde{B}}_{i}^{\dagger} \hat{\tilde{B}}_{i}+\left(\hbar \tilde{\lambda}_{i} \hat{B}_{0}^{\dagger} \hat{\tilde{B}}_{i}+\text { H.c. }\right)\right],
$$

where $\Omega_{0}=\sum_{\mathbf{k}}\left|g_{\mathbf{k}}\right|^{2} \omega_{\mathbf{k}} / G_{0}^{2}$ is the reaction coordinate frequency, and where the operators fulfill canonical commutation relations, $\left[\hat{\tilde{B}}_{i}, \hat{\tilde{B}}_{j}^{\dagger}\right]=\delta_{i j}$ and $\left[\hat{B}_{0}, \hat{\tilde{B}}_{i}^{\dagger}\right]=0$. Such a transformation is described within the theory of reaction coordinate mappings [39-43]. This form of $\hat{H}_{\mathrm{x}}$ describes the interaction of the exciton reaction coordinate $\hat{B}_{0}$ with an environment of residual exciton modes $\hat{\tilde{B}}_{i}$ through the coupling coefficients $\tilde{\lambda}_{i}$, as depicted in Fig. 2(b). By identifying the residual exciton modes, we can calculate the decay rate into the residual environment in the Markovian limit and assess the conditions under which the excitons can be described in terms of a single reaction coordinate. The properties of the residual environment are contained in the residual spectral density, $J_{\text {res }}(\omega)=\sum_{i>0}\left|\tilde{\lambda}_{i}\right|^{2} \delta\left(\omega-\tilde{\Omega}_{i}\right)$, which is related to the exciton spectral density $J(\omega)=\sum_{\mathbf{k}}\left|g_{\mathbf{k}}\right|^{2} \delta\left(\omega-\omega_{\mathbf{k}}\right)$ as $[44,45]$

$$
J_{\text {res }}(\omega)=\frac{G_{0}^{2} J(\omega)}{\Phi^{2}(\omega)+\pi^{2} J^{2}(\omega)},
$$

where $\Phi(\omega)=\lim _{\ell \rightarrow 0^{+}} \int_{\omega_{0}}^{\infty} d z J(z) \frac{\omega-z}{(\omega-z)^{2}+\ell^{2}}$.

To quantify the impact of the optical confinement geometry, we now assume that the electromagnetic field profile is separable in the lateral and out-of-plane coordinates and that the field is uniformly polarized with polarization vector $\mathbf{n}$, such that $\tilde{\mathbf{F}}_{\mathbf{c}}(\mathbf{r}, z)=\mathbf{n} F_{z}(z) F_{\|}(\mathbf{r})$. In this case, the outof-plane confinement length takes the intuitive form $L_{z}=$ $\epsilon_{\text {eff }} \int d z\left|F_{z}(z)\right|^{2} /\left|F_{z}\left(z_{0}\right)\right|^{2}$, where $\epsilon_{\text {eff }}$ is an effective dielectric constant accounting for the dielectric environment. In this way, $L_{z}$ can be formally separated from the lateral field distribution. Furthermore, when taking the in-plane field distribution to be Gaussian with confinement length $L ; F_{\|}(\mathbf{r})=$ $e^{-r^{2} /\left(2 L^{2}\right)} / \sqrt{\pi L^{2}}$, we can evaluate the spectral densities analytically as [37]

$$
\begin{aligned}
J(\omega) & =\Theta\left(\omega-\omega_{0}\right) J_{0} e^{-\left(\omega-\omega_{0}\right) / \xi}, \\
J_{\text {res }}(\omega) & =\Theta\left(\omega-\omega_{0}\right) \xi e^{\left(\omega-\omega_{0}\right) / \xi}\left\{\operatorname{Ei}^{2}\left[\left(\omega-\omega_{0}\right) / \xi\right]+\pi^{2}\right\}^{-1},
\end{aligned}
$$

where $\Theta$ is the Heaviside function, $\xi=\hbar /\left(2 M L^{2}\right)$ is a cutoff frequency, $J_{0}=G_{0}^{2} / \xi$ determines the overall magnitude of the spectral density and Ei denotes the exponential integral; these spectral densities are shown in Fig. 2(c). The overall scaling of the residual spectral density is determined by $\xi \propto L^{-2}$, which shows that the residual excitons are most important when the lateral confinement length is small.

To assess the limits of the model based on a single reaction coordinate, we investigate the conditions for neglecting the residual excitons. To this end, we study the linear response limit, where the nonlinear interactions $\hat{W}$ can be ignored and

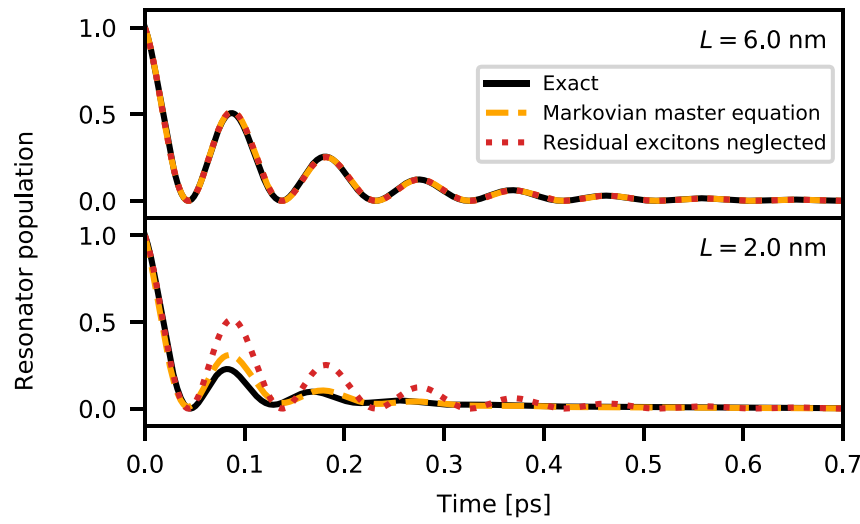

FIG. 3. (a) Exact time evolution of resonator excitation number, $\left\langle\hat{a}_{\mathrm{c}}^{\dagger} \hat{a}_{\mathrm{c}}\right\rangle$ (black solid lines), compared with the Markovian theory (orange dashed lines) and with the residual excitons ignored (red dotted lines) for a separable field profile with Gaussian in-plane distributions and different lateral confinement lengths, $L$. The 2D material was taken to be $\mathrm{WS}_{2}$, and $L_{z}=150 \mathrm{~nm}, \omega_{\mathrm{c}}=\Omega_{0}, \hbar \gamma_{\mathrm{c}}=5 \mathrm{meV}$, and $\left|\mathbf{n} \cdot \mathbf{p}_{\mathrm{cv}}\right| / p_{\mathrm{cv}}=0.5$. The light-matter coupling with these parameters is $\hbar G_{0}=22 \mathrm{meV}$.

consider the time evolution of a single resonator excitation. The excitation number $\left\langle\hat{a}_{\mathrm{c}}^{\dagger}(t) \hat{a}_{\mathrm{c}}(t)\right\rangle$ can be calculated exactly through the equation for the resonator amplitude $\phi_{\mathrm{c}}(t)$ [46],

$$
\frac{d \phi_{\mathrm{c}}(t)}{d t}=-\int_{0}^{t} K\left(t-t^{\prime}\right) \phi_{\mathrm{c}}\left(t^{\prime}\right) d t^{\prime}-\gamma_{\mathrm{c}} \phi_{\mathrm{c}}(t),
$$

where $K(\tau)=\Theta(\tau) \int d \omega J(\omega) e^{-i\left(\omega-\omega_{\mathrm{c}}\right) \tau}$ is a memory kernel, which fully accounts for interactions with the excitons, and $\gamma_{c}$ is the decay rate of the electromagnetic field. The excitation number of the resonator is then given by $\left\langle\hat{a}_{\mathrm{c}}^{\dagger}(t) \hat{a}_{\mathrm{c}}(t)\right\rangle=$ $\left|\phi_{\mathrm{c}}(t)\right|^{2}$. This time evolution is shown in Fig. 3 (black solid). As an alternative approach, we have derived a master equation for the reduced density operator of the resonant field and exciton reaction coordinate, in which the effect of the residual excitons is approximated by a Markovian decay with rate $\Gamma_{\text {res }}=2 \pi J_{\text {res }}\left(\omega_{+}\right)$, where $\omega_{+}=\left[\omega_{\mathrm{c}}+\Omega_{0}+\right.$ $\left.\sqrt{4 G_{0}^{2}+\left(\omega_{\mathrm{c}}-\Omega_{0}\right)^{2}}\right] / 2$ is the frequency of the upper polariton [37]. The residual decay rate is shown in Fig. 1(b) for $\omega_{\mathrm{c}}=\Omega_{0}$, and the time evolution generated by this master equation is shown in Fig. 3 (orange dashed) along with the result obtained when ignoring the residual excitons entirely (red dotted). This shows that the residual exciton environment starts to play a role for $L$ below $\sim 4 \mathrm{~nm}$, and that it is well approximated by the Markovian theory [37].

We now proceed to study the impact of the nonlinear exciton-exciton interactions and the prospects for reaching the polariton blockade regime. To do so, we assume that the residual exciton modes, $\hat{\tilde{B}}_{i}$, are weakly populated, such that the exciton-exciton interaction, $\hat{W}$, is only significant within the reaction coordinate. This assumption is reasonable in the limit where the exciton reaction coordinate is decoupled from the residual exciton modes and therefore is the only exciton mode with appreciable population. The interaction is thus approximated as $\hat{W} \approx \hat{W}_{0}:=\hbar W_{0}^{\prime} \hat{B}_{0}^{\dagger} \hat{B}_{0}^{\dagger} \hat{B}_{0} \hat{B}_{0}$. Assuming $L \gg a_{\mathrm{B}}$, we can neglect the momentum dependence of the matrix element $W_{\mathbf{k k} \mathbf{k}^{\mathbf{q}}}$ [31], such that the interaction strength within the 
reaction coordinate becomes $W_{0}^{\prime} \simeq S W_{000} \eta_{\mathbf{n}}\left[\int d^{2} \mathbf{r} \mid \tilde{\mathbf{F}}_{\mathrm{c}}\left(\mathbf{r}, z_{0}\right)\right.$. $\left.\left.\mathbf{p}_{\mathrm{cv}}\right|^{2}\right]^{-2} \int d^{2} \mathbf{r}\left|\tilde{\mathbf{F}}_{\mathrm{c}}\left(\mathbf{r}, z_{0}\right) \cdot \mathbf{p}_{\mathrm{cv}}\right|^{4}[13,37]$. This quantity determines the nonlinear energy shift of the exciton reaction coordinate. Here, the polarization-dependent prefactor $\eta_{\mathbf{n}}$ takes values between $1 / 2$ (reached in the limit of a linearly polarized resonator mode) and 1 (reached in the limit of a circularly polarized resonator mode) [37]. In the calculations presented here, we take $\eta_{\mathbf{n}}=1$, corresponding to a circularly polarized mode.

The zero-momentum interaction matrix element has previously been found to be well approximated from the binding energy $\left(E_{\mathrm{b}}\right)$ and Bohr radius as $\hbar W_{000} \simeq \alpha E_{\mathrm{b}} a_{\mathrm{B}}^{2} / S$. Using $\alpha=2.07$ for $\mathrm{WS}_{2}$ [47], this leads to $\hbar S W_{000} \simeq 2.04 \mathrm{eVnm}^{2}$. For the Gaussian field we find $\hbar W_{0}^{\prime}=\alpha E_{\mathrm{b}} a_{\mathrm{B}}^{2} /\left(2 \pi L^{2}\right)$, which scales as the inverse mode area. In contrast to the case of a laterally nanostructured 2D material $[48,49]$, the nonlinear interaction strength here is entirely determined by the lateral confinement of the resonant field and the exciton Coulomb interaction strength. Within the master equation formalism, we can account for nonradiative exciton decay and dephasing due to phonon interactions [50] through a nonradiative exciton decay rate $\gamma_{x}$, and dephasing rate $\gamma_{x}^{\prime}$, which both increase with temperature [37,51].

To investigate polariton blockade and single-photon nonlinearities, we consider the second-order correlation function $g^{(2)}(\tau)=\left\langle\hat{a}_{\mathrm{c}}^{\dagger} \hat{a}_{\mathrm{c}}^{\dagger}(\tau) \hat{a}_{\mathrm{c}}(\tau) \hat{a}_{\mathrm{c}}\right\rangle /\left\langle\hat{a}_{\mathrm{c}}^{\dagger} \hat{a}_{\mathrm{c}}\right\rangle^{2}$ [52], evaluated with respect to the steady-state density operator. Zero-delay values of $g^{(2)}(0)<1$ are a signature of polariton blockade, and $g^{(2)}(0)$ reaches 0 in the case of perfect blockade [13,53]. It has previously been shown that in the absence of dephasing, near-zero values of $g^{(2)}(0)$ can be reached, even in the limit of a small nonlinear interaction, $W_{0}^{\prime} \ll \gamma_{\mathrm{x}}[54,55]$. This is an unconventional polariton blockade effect, which arises due to destructive interference between the excitation paths leading to excitation of multiple polaritons [56]. We note that the original theoretical descriptions of unconventional polariton blockade $[54,56]$ found the effect in a system of two coupled electromagnetic resonators, with at least one of them being nonlinear. In contrast, the two coupled modes in the present situation are constituted by the resonator and the exciton reaction coordinate, where only the latter is nonlinear. We use the Markovian master equation to evaluate the minimal value of $g^{(2)}(0)$ that can be reached for a given lateral confinement length $L$, of the resonator with a Gaussian electromagnetic field profile, driven by a continuous-wave laser field with frequency $\omega_{\mathrm{d}}$ and amplitude $F$. This driving is described by adding the term $F e^{+i \omega_{\mathrm{d}} t} \hat{a}_{\mathrm{c}}+F^{*} e^{-i \omega_{\mathrm{d}} t} \hat{a}_{\mathrm{c}}^{\dagger}$ to $\hat{H}_{\mathrm{c}}$. The nonlinear interaction is accounted for within the exciton reaction coordinate through the Hamiltonian contribution $\hat{W}_{0}$. We then numerically minimize $g^{(2)}(0)$ with respect to $\omega_{\mathrm{d}}$ and $\omega_{\mathrm{c}}$ for each parameter setting.

Figure 4(a) shows the minimum attainable $g^{(2)}(0)$ for $\mathrm{WS}_{2}$ as a function of $L$ at cryogenic temperature (blue colors) and room temperature (red colors), and for different experimentally relevant resonator linewidths $\gamma_{\mathrm{c}}$. In Fig. 4(b), we show the nonlinear shift $W_{0}^{\prime}$ as a function of $L$ and show the total intrinsic exciton linewidth $\Gamma_{\mathrm{x}}=\gamma_{\mathrm{x}}+\gamma_{\mathrm{x}}^{\prime}$, for the two temperature settings, along with the dephasing contribution to the linewidth, $\gamma_{x}^{\prime}$. From these calculations, we observe the
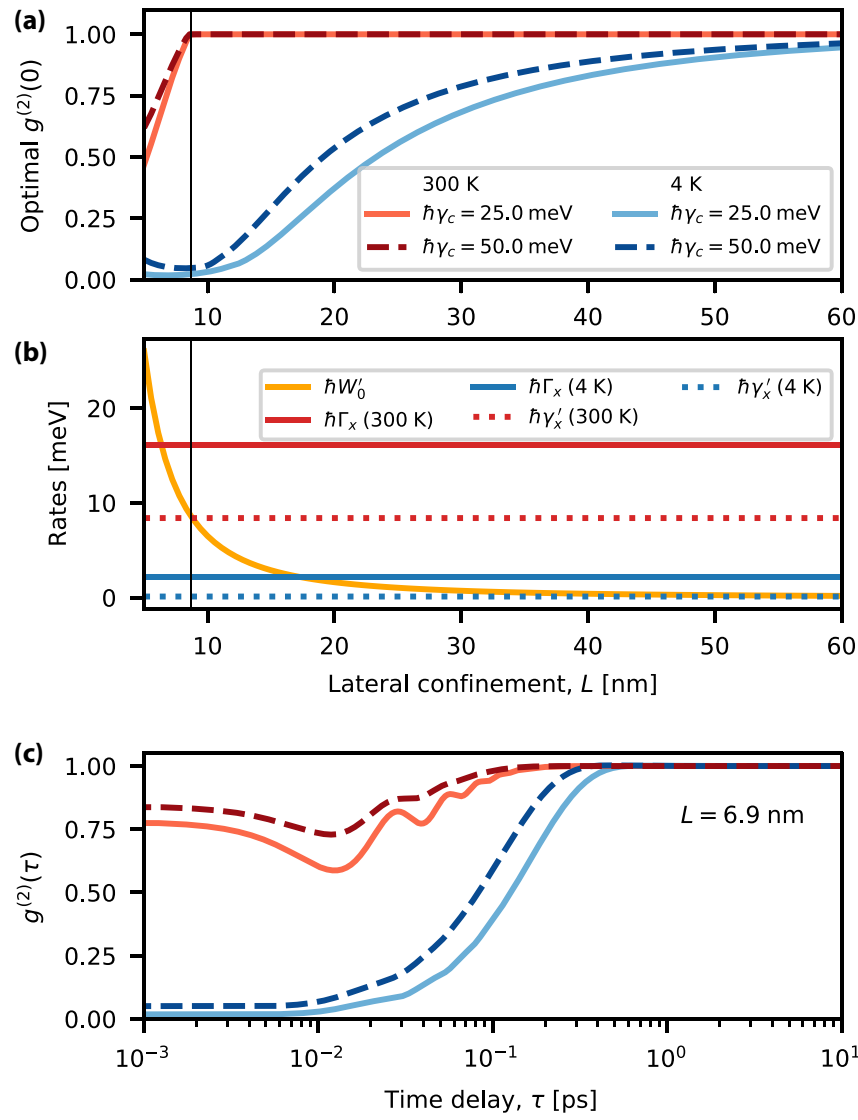

FIG. 4. (a) Optimal antibunching vs lateral confinement, $L$, for monolayer $\mathrm{WS}_{2}$ coupled to a resonant field with Gaussian inplane distribution at temperatures of 4 and $300 \mathrm{~K}$ and resonator linewidths $\hbar \gamma_{\mathrm{c}}$ of 25 and $50 \mathrm{meV}$. Parameters: $L_{z}=50 \mathrm{~nm}, F=$ $1.5 \mathrm{meV},\left|\mathbf{p}_{\mathrm{cv}} \cdot \mathbf{n}\right| / p_{\mathrm{cv}}=0.75$, corresponding to $\hbar G_{0}=57.5 \mathrm{meV}$. (b) Nonlinear interaction strength $W_{0}^{\prime}$ vs $L$, compared to exciton linewidth, $\Gamma_{\mathrm{x}}=\gamma_{\mathrm{x}}+\gamma_{\mathrm{x}}^{\prime}$, and exciton dephasing, $\gamma_{\mathrm{x}}^{\prime}$. The thin vertical lines in panels a and b mark the value of $L$ where $g^{(2)}(0)$ drops below 1 for $T=300 \mathrm{~K}$. (c) Delay-time dependence of steady-state secondorder correlation function for optimized parameters as in panel a, at a lateral confinement length of $L=6.9 \mathrm{~nm}$. Plot signatures correspond to those in panel a.

important result that reductions in $g^{(2)}(0)$ below the classical limit $g^{(2)}(0)<1$ become feasible when $W_{0}^{\prime}$ overcomes the polariton dephasing $\gamma_{x}^{\prime}$, whereas it does not need to overcome the total linewidth. This follows from the difference between the behavior at the two temperatures. For $T=4 \mathrm{~K}, W_{0}^{\prime}$ is above $\gamma_{\mathrm{x}}^{\prime}$ for $L \lesssim 75 \mathrm{~nm}$ and $g^{(2)}(0)$ increases smoothly from near-zero values towards 1 . At $T=300 \mathrm{~K}, W_{0}^{\prime}$ exceeds $\gamma_{\mathrm{x}}^{\prime}$ for $L \lesssim 9 \mathrm{~nm}$, at which point the optimal $g^{(2)}(0)$ starts to decrease from unity abruptly, due to the steep increase in $W_{0}^{\prime}$ as $L$ is decreased (indicated with thin vertical lines in Figs. 4(a) and 4(b). In this way, we identify excitonic dephasing as the main challenge for reaching the blockade regime, and we interpret this limitation as originating from a decoherenceinduced suppression of the destructive interference between two-polariton exciton paths, which is responsible for polariton blockade. In Fig. 4(c), we show the delay-time dependence of $g^{(2)}(\tau)$, as calculated from the master equation using the quantum regression theorem [57], which transitions from its 
minimal value at $\tau=0$ towards unity on a time scale on the order of $1 \mathrm{ps}$. The antibunching appearing on this picosecond time scale should be observable with established ultrafast detection techniques [58-60]. Due to a significant detuning on the order of 100-300 meV between the resonant field and the exciton in the two-frequency optimized configuration, the signal does not exhibit Rabi oscillations as reported in previous studies on unconventional polariton blockade [54-56]. This significant detuning follows from the fact that our optimization procedure does not impose constraints on the frequencies $\omega_{\mathrm{c}}$ and $\omega_{\mathrm{d}}$, whereas previous studies have fixed one of these detunings in the optimization procedure.

We have presented a microscopic theory of light-matter interaction in electromagnetic resonators coupled to pristine sheets of 2D semiconductor, whereby we unambiguously and analytically identify all relevant dynamical parameters in terms of fundamental material parameters. We find that rather than the mode volume $\sim L^{2} L_{z}$, it is the out-of plane confinement length $L_{z}$ that controls the light-matter interaction, while the lateral length $L$ determines both the nonlinear interaction and coupling to residual modes. Using this theory, we have identified a significant operational window of lateral length scales, where the nonlinear exciton response is large enough to enable a pronounced polariton blockade while the coupling to residual modes can be neglected. Our calculations show that lateral dimensions of the order of $40 \mathrm{~nm}$ or smaller are required to see significant antibunching, $g^{(2)}(0)<1 / 2$, at cryogenic temperatures, whereas smaller dimensions of around $10 \mathrm{~nm}$ are required at room temperature. Such dimensions are well within the limits of contemporary nanofabrication [61] using either plasmonic resonators $[62,63]$ or optical cavities with extreme dielectric confinement of light $[64,65]$.

The authors thank Peder Meisner Lyngby for valuable discussions. This work was supported by the Danish National Research Foundation through NanoPhoton - Center for Nanophotonics, Grant No. DNRF147 and Center for Nanostructured Graphene, Grant No. DNRF103. NS acknowledges support from the Villum Foundation through Grant No. 00028233. MW and NS acknowledge support from the Independent Research Fund Denmark - Natural Sciences (Project no. 0135-00403B). EVD acknowledges support from Independent Research Fund Denmark through an International Postdoc fellowship (Grant No. 0164-00014B).
[1] J. Wen, H. Wang, W. Wang, Z. Deng, C. Zhuang, Y. Zhang, F. Liu, J. She, J. Chen, H. Chen, S. Deng, and N. Xu, Roomtemperature strong light-matter interaction with active control in single plasmonic nanorod coupled with two-dimensional atomic crystals, Nano Lett. 17, 4689 (2017).

[2] D. Zheng, S. Zhang, Q. Deng, M. Kang, P. Nordlander, and $\mathrm{H}$. Xu, Manipulating coherent plasmon-exciton interaction in a single silver nanorod on monolayer $\mathrm{WSe}_{2}$, Nano Lett. 17, 3809 (2017).

[3] M.-E. Kleemann, R. Chikkaraddy, E. M. Alexeev, D. Kos, C. Carnegie, W. Deacon, A. C. De Pury, C. Große, B. de Nijs, J. Mertens, A. I. Tartakovskii, and J. J. Baumberg, Strongcoupling of $\mathrm{WSe}_{2}$ in ultra-compact plasmonic nanocavities at room temperature, Nat. Commun. 8, 1296 (2017).

[4] J. Cuadra, D. G. Baranov, M. Wersäll, R. Verre, T. J. Antosiewicz, and T. Shegai, Observation of tunable charged exciton polaritons in hybrid monolayer $\mathrm{WS}_{2}$-plasmonic nanoantenna system, Nano Lett. 18, 1777 (2018).

[5] M. Stührenberg, B. Munkhbat, D. G. Baranov, J. Cuadra, A. B. Yankovich, T. J. Antosiewicz, E. Olsson, and T. Shegai, Strong light-matter coupling between plasmons in individual gold bipyramids and excitons in mono-and multilayer $\mathrm{WSe}_{2}, \mathrm{Nano}$ Lett. 18, 5938 (2018).

[6] X. Han, K. Wang, X. Xing, M. Wang, and P. Lu, Rabi splitting in a plasmonic nanocavity coupled to a $\mathrm{WS}_{2}$ monolayer at room temperature, ACS Photonics 5, 3970 (2018).

[7] M. Geisler, X. Cui, J. Wang, T. Rindzevicius, L. Gammelgaard, B. S. Jessen, P. A. D. Goncalves, F. Todisco, P. Bøggild, A. Boisen, M. Wubs, N. A. Mortensen, S. Xiao, and N. Stenger, Single-crystalline gold nanodisks on $\mathrm{WS}_{2}$ mono-and multilayers for strong coupling at room temperature, ACS Photonics 6 , 994 (2019).
[8] J. Qin, Y.-H. Chen, Z. Zhang, Y. Zhang, R. J. Blaikie, B. Ding, and M. Qiu, Revealing Strong Plasmon-Exciton Coupling Between Nanogap Resonators and Two-Dimensional Semiconductors at Ambient Conditions, Phys. Rev. Lett. 124, 063902 (2020).

[9] D. Sanvitto and S. Kéna-Cohen, The road towards polaritonic devices, Nat. Mater. 15, 1061 (2016).

[10] J. P. Karr, A. Baas, R. Houdré, and E. Giacobino, Squeezing in semiconductor microcavities in the strong-coupling regime, Phys. Rev. A 69, 031802(R) (2004).

[11] T. Boulier, M. Bamba, A. Amo, C. Adrados, A. Lemaitre, E. Galopin, I. Sagnes, J. Bloch, C. Ciuti, E. Giacobino, and A. Bramati, Polariton-generated intensity squeezing in semiconductor micropillars, Nat. Commun. 5, 3260 (2014).

[12] A. Imamoğlu, R. J. Ram, S. Pau, and Y. Yamamoto, Nonequilibrium condensates and lasers without inversion: Excitonpolariton lasers, Phys. Rev. A 53, 4250 (1996).

[13] A. Verger, C. Ciuti, and I. Carusotto, Polariton quantum blockade in a photonic dot, Phys. Rev. B 73, 193306 (2006).

[14] S. Ferretti and D. Gerace, Single-photon nonlinear optics with Kerr-type nanostructured materials, Phys. Rev. B 85, 033303 (2012).

[15] A. Delteil, T. Fink, A. Schade, S. Höfling, C. Schneider, and A. İmamoğlu, Towards polariton blockade of confined excitonpolaritons, Nat. Mater. 18, 219 (2019).

[16] G. Muñoz-Matutano, A. Wood, M. Johnsson, X. Vidal, B. Q. Baragiola, A. Reinhard, A. Lemaître, J. Bloch, A. Amo, G. Nogues, B. Besga, M. Richard, and T. Volz, Emergence of quantum correlations from interacting fibre-cavity polaritons, Nat. Mater. 18, 213 (2019).

[17] D. E. Chang, A. S. Sørensen, E. A. Demler, and M. D. Lukin, A single-photon transistor using nanoscale surface plasmons, Nat. Phys. 3, 807 (2007). 
[18] T. Volz, A. Reinhard, M. Winger, A. Badolato, K. J. Hennessy, E. L. Hu, and A. Imamoğlu, Ultrafast all-optical switching by single photons, Nat. Photonics 6, 605 (2012).

[19] J. Hwang, M. Pototschnig, R. Lettow, G. Zumofen, A. Renn, S. Götzinger, and V. Sandoghdar, A single-molecule optical transistor, Nature (London) 460, 76 (2009).

[20] S. Wu, S. Buckley, A. M. Jones, J. S. Ross, N. J. Ghimire, J. Yan, D. G. Mandrus, W. Yao, F. Hatami, J. Vučković, A. Majumdar, and $\mathrm{X}$. Xu, Control of two-dimensional excitonic light emission via photonic crystal, 2D Mater. 1, 011001 (2014).

[21] Y. J. Noori, Y. Cao, J. Roberts, C. Woodhead, R. BernardoGavito, P. Tovee, and R. J. Young, Photonic crystals for enhanced light extraction from 2D materials, ACS Photonics 3, 2515 (2016).

[22] T. K. Fryett, K. L. Seyler, J. Zheng, C.-H. Liu, X. Xu, and A. Majumdar, Silicon photonic crystal cavity enhanced secondharmonic generation from monolayer $\mathrm{WSe}_{2}, 2 \mathrm{D}$ Mater. 4, 015031 (2016).

[23] S. Hu and S. M. Weiss, Design of photonic crystal cavities for extreme light concentration, ACS Photonics 3, 1647 (2016).

[24] H. Choi, M. Heuck, and D. Englund, Self-Similar Nanocavity Design with Ultrasmall Mode Volume for Single-Photon Nonlinearities, Phys. Rev. Lett. 118, 223605 (2017).

[25] F. Wang, R. E. Christiansen, Y. Yu, J. Mørk, and O. Sigmund, Maximizing the quality factor to mode volume ratio for ultrasmall photonic crystal cavities, Appl. Phys. Lett. 113, 241101 (2018).

[26] C. Tserkezis, A. I. Fernández-Domínguez, P. Gonçalves, F. Todisco, J. D. Cox, K. Busch, N. Stenger, S. I. Bozhevolnyi, N. A. Mortensen, and C. Wolff, on the applicability of quantumoptical concepts in strong-coupling nanophotonics, Rep. Prog. Phys. 83, 082401 (2020).

[27] S. Latini, E. Ronca, U. De Giovannini, H. Hübener, and A. Rubio, Cavity control of excitons in two-dimensional materials, Nano Lett. 19, 3473 (2019).

[28] O. Kyriienko, D. N. Krizhanovskii, and I. A. Shelykh, Nonlinear Quantum Optics with Trion Polaritons in 2D Monolayers: Conventional and Unconventional Photon Blockade, Phys. Rev. Lett. 125, 197402 (2020).

[29] T. Usui, Excitations in a high density electron gas. I, Prog. Theor. Phys. 23, 787 (1960).

[30] E. Hanamura, Theory of the high density exciton. I, J. Phys. Soc. Jpn. 29, 50 (1970).

[31] F. Tassone and Y. Yamamoto, Exciton-exciton scattering dynamics in a semiconductor microcavity and stimulated scattering into polaritons, Phys. Rev. B 59, 10830 (1999).

[32] G. Rochat, C. Ciuti, V. Savona, C. Piermarocchi, A. Quattropani, and P. Schwendimann, Excitonic bloch equations for a two-dimensional system of interacting excitons, Phys. Rev. B 61, 13856 (2000).

[33] T. Cheiwchanchamnangij and W. R. L. Lambrecht, Quasiparticle band structure calculation of monolayer, bilayer, and bulk $\mathrm{MoS}_{2}$, Phys. Rev. B 85, 205302 (2012).

[34] G. Wang, A. Chernikov, M. M. Glazov, T. F. Heinz, X. Marie, T. Amand, and B. Urbaszek, Colloquium: Excitons in atomically thin transition metal dichalcogenides, Rev. Mod. Phys. 90, 021001 (2018).

[35] T. Olsen, S. Latini, F. Rasmussen, and K. S. Thygesen, Simple Screened Hydrogen Model of Excitons in Two-Dimensional Materials, Phys. Rev. Lett. 116, 056401 (2016).
[36] S. Franke, S. Hughes, M. K. Dezfouli, P. T. Kristensen, K. Busch, A. Knorr, and M. Richter, Quantization of Quasinormal Modes for Open Cavities and Plasmonic Cavity Quantum Electrodynamics, Phys. Rev. Lett. 122, 213901 (2019).

[37] E. V. Denning, M. Wubs, N. Stenger, J. Mørk, and P. T. Kristensen, Quantum theory of two-dimensional materials coupled to electromagnetic resonators, Phys. Rev. B 105, 085306 (2022).

[38] S. Stobbe, P. T. Kristensen, J. E. Mortensen, J. M. Hvam, J. Mørk, and P. Lodahl, Spontaneous emission from large quantum dots in nanostructures: Exciton-photon interaction beyond the dipole approximation, Phys. Rev. B 86, 085304 (2012).

[39] A. J. Leggett, Quantum tunneling in the presence of an arbitrary linear dissipation mechanism, Phys. Rev. B 30, 1208 (1984).

[40] A. Garg, J. N. Onuchic, and V. Ambegaokar, Effect of friction on electron transfer in biomolecules, J. Chem. Phys. 83, 4491 (1985).

[41] K. H. Hughes, C. D. Christ, and I. Burghardt, Effective-mode representation of non-Markovian dynamics: A hierarchical approximation of the spectral density. I. Application to single surface dynamics, J. Chem. Phys. 131, 024109 (2009).

[42] K. H. Hughes, C. D. Christ, and I. Burghardt, Effective-mode representation of non-Markovian dynamics: A hierarchical approximation of the spectral density. II. Application to environment-induced nonadiabatic dynamics, J. Chem. Phys. 131, 124108 (2009).

[43] J. Iles-Smith, A. G. Dijkstra, N. Lambert, and A. Nazir, Energy transfer in structured and unstructured environments: Master equations beyond the Born-Markov approximations, J. Chem. Phys. 144, 044110 (2016).

[44] R. Martinazzo, B. Vacchini, K. H. Hughes, and I. Burghardt, Communication: Universal Markovian reduction of Brownian particle dynamics, J. Chem. Phys. 134, 011101 (2011).

[45] M. P. Woods, R. Groux, A. W. Chin, S. F. Huelga, and M. B. Plenio, Mappings of open quantum systems onto chain representations and Markovian embeddings, J. Math. Phys. 55, 032101 (2014).

[46] N. Vats, S. John, and K. Busch, Theory of fluorescence in photonic crystals, Phys. Rev. A 65, 043808 (2002).

[47] V. Shahnazaryan, I. Iorsh, I. A. Shelykh, and O. Kyriienko, Exciton-exciton interaction in transition-metal dichalcogenide monolayers, Phys. Rev. B 96, 115409 (2017).

[48] H.-X. Wang, A. Zhan, Y.-D. Xu, H.-Y. Chen, W.-L. You, A. Majumdar, and J.-H. Jiang, Quantum many-body simulation using monolayer exciton-polaritons in coupled-cavities, J. Phys.: Condens. Matter 29, 445703 (2017).

[49] A. Ryou, D. Rosser, A. Saxena, T. Fryett, and A. Majumdar, Strong photon antibunching in weakly nonlinear two-dimensional exciton-polaritons, Phys. Rev. B 97, 235307 (2018).

[50] G. Moody, C. K. Dass, K. Hao, C.-H. Chen, L.-J. Li, A. Singh, K. Tran, G. Clark, X. Xu, G. Berghäuser, E. Malic, A. Knorr, and $\mathrm{X}$. Li, Intrinsic homogeneous linewidth and broadening mechanisms of excitons in monolayer transition metal dichalcogenides, Nat. Commun. 6, 8315 (2015).

[51] M. Selig, G. Berghäuser, A. Raja, P. Nagler, C. Schüller, T. F. Heinz, T. Korn, A. Chernikov, E. Malic, and A. Knorr, Exci- 
tonic linewidth and coherence lifetime in monolayer transition metal dichalcogenides, Nat. Commun. 7, 13279 (2016).

[52] R. Hanbury Brown and R. Q. Twiss, Correlation between photons in two coherent beams of light, Nature (London) 177, 27 (1956).

[53] C. Gardiner, P. Zoller, and P. Zoller, Quantum Noise: A Handbook of Markovian and Non-Markovian Quantum Stochastic Methods with Applications to Quantum Optics (Springer Science \& Business Media, Berlin, 2004).

[54] T. C. H. Liew and V. Savona, Single Photons from Coupled Quantum Modes, Phys. Rev. Lett. 104, 183601 (2010).

[55] S. Ferretti, V. Savona, and D. Gerace, Optimal antibunching in passive photonic devices based on coupled nonlinear resonators, New J. Phys. 15, 025012 (2013).

[56] M. Bamba, A. Imamoğlu, I. Carusotto, and C. Ciuti, Origin of strong photon antibunching in weakly nonlinear photonic molecules, Phys. Rev. A 83, 021802(R) (2011).

[57] H.-P. Breuer and F. Petruccione, The Theory of Open Quantum Systems (Oxford University Press, Oxford, 2002).

[58] D. F. McAlister and M. G. Raymer, Ultrafast photon-number correlations from dual-pulse, phase-averaged homodyne detection, Phys. Rev. A 55, R1609(R) (1997).

[59] M. Aßmann, F. Veit, M. Bayer, M. van der Poel, and J. M. Hvam, Higher-order photon bunching in a semiconductor microcavity, Science 325, 297 (2009).

[60] M. Aßmann, F. Veit, J.-S. Tempel, T. Berstermann, H. Stolz,
M. van der Poel, J. M. Hvam, and M. Bayer, Measuring the dynamics of second-order photon correlation functions inside a pulse with picosecond time resolution, Opt. Express 18, 20229 (2010).

[61] B. S. Jessen, L. Gammelgaard, M. R. Thomsen, D. M. A. Mackenzie, J. D. Thomsen, J. M. Caridad, E. Duegaard, K. Watanabe, T. Taniguchi, T. J. Booth, T. G. Pedersen, A.-P. Jauho, and P. Bøggild, Lithographic band structure engineering of graphene, Nat. Nanotechnol. 14, 340 (2019).

[62] H. Duan, A. I. Fernández-Domínguez, M. Bosman, S. A. Maier, and J. K. W. Yang, Nanoplasmonics: classical down to the nanometer scale, Nano Lett. 12, 1683 (2012).

[63] F. Benz, M. K. Schmidt, A. Dreismann, R. Chikkaraddy, Y. Zhang, A. Demetriadou, C. Carnegie, H. Ohadi, B. de Nijs, R. Esteban, J. Aizpurua, and J. J. Baumberg, Singlemolecule optomechanics in "picocavities", Science 354, 726 (2016).

[64] S. Hu, M. Khater, R. Salas-Montiel, E. Kratschmer, S. Engelmann, W. M. J. Green, and S. M. Weiss, Experimental realization of deep-subwavelength confinement in dielectric optical resonators, Sci. Adv. 4, eaat235 (2018).

[65] M. Albrechtsen, B. V. Lahijani, R. E. Christiansen, V. T. H. Nguyen, L. N. Casses, S. E. Hansen, N. Stenger, O. Sigmund, H. Jansen, J. Mørk, and S. Stobbe, Nanometer-scale photon confinement inside dielectrics, arXiv:2108.01681 (2021). 